\title{
THE MOST COMMONLY USED METHODS OF TREATMENT FOR BRUXISM - A LITERATURE REVIEW
}

\author{
Paulina Wilmont, Klara Saczuk, Łukasz Pawlak, Monika Łukomska-Szymańska \\ Department of General Dentistry, Medical University of Lodz, Poland
}

\begin{abstract}
Bruxism is referred to as grinding and clenching of the teeth. It is a disorder affecting an increasing number of patients. This disease causes severe ailments that negatively influencing the daily functioning and manifesting, among the others, as toothaches, masticatory muscle pain, headaches, and tooth hypersensitivity.

The aim of the work is to analyse the contemporary treatment methods and methods of alleviating the effects of the disease. In addition, it evaluates the usefulness of these methods in daily dental practice. The article shows the contemporary view of treatment, including the multidisciplinary approach. The authors present treatment methods of bruxism, such as: splints, physiotherapy, psychotherapy, biofeedback, and pharmacological treatment. The variety of methods currently used and the lack of clear guidelines for treatment of bruxism should be emphasised. The article also draws attention to the need to modify the daily behaviour of the patient through relaxation exercises or biofeedback, in order to eliminate bruxism and not just alleviate its symptoms.

A multidirectional approach and treatment of bruxism can produce satisfactory and lasting results.
\end{abstract}

KEY WORDS: bruxism, treatment, physiotherapy, biofeedback, psychotherapy.

J Stoma 2018; 71, 4: 350-355

DOI: https://doi.org/10.5114/jos.2018.83409

\section{INTRODUCTION}

In the past, bruxism was referred to as a parafunction involving grinding and clenching of the teeth, mainly at night (nocturnal bruxism). In 2012, a group of experts from around the world dealing with the subject of bruxism defined it as "a repetitive jaw-muscle activity characterised by clenching or grinding of the teeth and/or by bracing or thrusting of the mandible" [1]. At the same time, these scientists question the definition of bruxism as a parafunction, because it indicates only negative causes, and they also suggested that bruxism episodes can also improve the airflow through the airways [1]. Regardless of the definition of bruxism, the associated disorders, such as pathological tooth wear, masticatory muscle pain, headaches, or toothaches (unconnected with any inflammatory process or tooth decay), are an increasingly frequent reason for patients to present to the dental office. Therefore, new effective treatment methods are needed. The management of bruxism is mainly based on treating or alleviating symptoms caused by increased force occurring while clenching the teeth.

\section{CURRENT VIEWS ON TREATMENT}

Most researchers underline the need for the multi-treatment of bruxism patients, including, as well as dental treatment (e.g. restorations), psychotherapy, physiotherapy, medication, and speech therapy [2-4]. Others indicate that there is no specific treatment for nocturnal bruxism,

\section{JOURNAL OF STOMATOLOGY CZASOPISMO STOMATOLOGICZNE}

AdDress For CORRESPONDENCE: Paulina Wilmont, Department of General Dentistry, Medical University of Lodz, 251 Pomorska St, 92-213 Lodz, Poland, phone: +48 4267574 61, fax: +48426757462, e-mail: paulina.wilmont@umed.lodz.pl 
but it can be eliminated through everyday behaviour modification, relaxation exercises, or biofeedback, while other methods merely serve to reduce the destructive impact of the disease [5]. The treatment plan should include, along with treating symptoms and reducing occlusal disorders, psychological and physical stress reduction and changing neuromuscular patterns [6].

\section{SPLINTS}

The basic method of treatment is the use of occlusal splints made of hard material. These appliances are aimed at muscle relaxation and protection of teeth from further damage [7-9]. Some authors describe decreased muscle tone demonstrated in EMG in about $50 \%$ of patients with occlusal splints. There are various types of splints, for example: maxillary occlusal splint (MOS), mandibular advancement device (MAD), canine protected hard stabilisation splint, Bruxogard soft splint, and others. Many studies have confirmed the effectiveness of splints in reducing the number of bruxism episodes per hour of sleep [10-13]. Singh et al. [14] proved MOS and MAD to be equally effective in improving the quality of sleep in respondents with bruxism, but a greater reduction in bruxism episodes per hour of sleep occurred in ones using MAD. Landry et al. [15] achieved similar results, showing a twofold decrease in that parameter. However, MAD use was associated with greater discomfort and thus resulted in a lower level of satisfaction than that seen in MOS users [7, 15]. Karakis et al. [13] showed no statistically significant changes in occlusal force after three and six weeks of the application of a canine protected hard stabilisation splint, but such a change occurred after six weeks of Bruxogard soft splint usage. However, when comparing using the craniomandibular index (CMI) showing the degree of dysfunction, muscle soreness on palpation, and pain around temporomandibular joints, the use of both splints made of hard or soft materials resulted in a significant improvement. The authors suggested that differences in the distribution of occlusal forces and reduced muscle fatigue can arise from different mechanisms of action of both splint types [13]. As early as in the 1990s, Wright et al. [16] and Pettengill et al. [17] indicated the usefulness of hard and soft splints in the short-term treatment of temporomandibular symptoms. Scopel et al. [18] showed regression of pain between four and nine weeks from the commencement of splint therapy. Other types of splints that, according to Giannakopoulos et al. [19], can be used in the immediate treatment of painful disorders of the temporomandibular joints are prefabricated oral splints with water-filled elastic pads (Aqualizer), vacuum-formed co-polyester oral splints, and Michigan-type hard splints. In that case, the use of hard stabilising splints is time-consuming due to the necessary engagement of a technical laboratory, and thus difficult to apply in immediate treatment. It was shown that all those types of splints caused a decrease in pain, but statistically significant effects of the treatment in a twoweek period were achieved by using vacuum-formed co-polyester oral splints. According to other researchers, hard splints, as well as soft ones, proved to be effective in long-term treatment [20]. However, that is not a perfect method, because episodes of grinding and clenching may re-intensify after the end of the treatment [7].

According to Gomes et al. [4], using only an occlusal splint did not significantly affect the electromyographic activity of the masseter and temporalis muscles; a combination of massage and splints yielded no improvement in EMG either (after a four-week treatment period). Nevertheless, regarding improvement in the intensity of temporomandibular disorders based on the Fonseca Patient History Index, the best results were found for a simultaneous combination of a massage and occlusal splint. The use of just one of those solutions or a silicone splint did not offer significant improvement. No change in electromyographic activity was reported in earlier studies by Weggen et al. [21] either. In contrast to those authors, Zhang et al. [22] achieved changes in EMG in their subjects after four weeks of treatment. Although the use of splints in patients with bruxism does not cure the condition, it was repeatedly demonstrated that it leads to a reduction in myofascial pain and improves the quality of life and the maximum opening of the jaw $[8,13,23-25]$. Some studies showed a reduction in the initial muscle electromyographic activity after the treatment with splints, followed, however, by a subsequent return to the initial level $[26,27]$, which may be due to muscle adaptation mechanisms [4].

Another type of splint used in the treatment of bruxism is a nociceptive trigeminal inhibition splint (NTI), placed between the upper central and lower incisors, resulting in immediate teeth disclusion during parafunctional activity [28]. The use of the splint causes immediate but short-term reduction in bioelectric potentials if the splint has a height of less than $6 \mathrm{~mm}$, but if the height is equal to or greater than $6 \mathrm{~mm}$, its use is followed by an immediate EMG increase [29-32]. Baad Hansen et al. [33] observed no changes in EMG after two weeks of NTI splint use.

Dalewski et al. [28] observed no significant changes in potentials in either of their patient groups (occlusal splint and NTI users). Neither the occlusal splint nor the NTI had a significant impact on the standardised muscle activity test results.

Another type of splint, successfully used in the discussed ailments, is the sublingual relaxation splint (SRS), which is a kind of relaxation splint for the lower dental arch. Gawriołek et al. [34] in their research used the S. Włoch method, which includes SRS, self-awareness training, and myorelaxation exercises. They obtained a decrease in pain in patients and a mandibular velocity in the range close to that of the control group, which indicates the usefulness of SRS in relieving symptoms of bruxism. 


\section{PHYSIOTHERAPY}

Physiotherapy plays an important role in the treatment of bruxism. It is used primarily to reduce its negative effects. Physiotherapeutic methods include, among others, massage, manual therapy, therapeutic exercises (including relaxation exercises), electrotherapy, muscular awareness therapy, moist heat, laser, and microwaves [35-44]. The massage of masticatory muscles serves, among others, to reduce pain and tension of the muscles, improve their blood circulation, and prevent the adherence of tissues $[8,36,45]$. Studies have shown no changes in EMG after that type of therapy $[4,26,27$, 47]. Electrotherapy methods include, among others: TENS (transcutaneous electrical nerve stimulation) and MENS (microcurrent electrical nerve stimulation). Rajpurohit et al. [39] examined both kinds of currents administered to patients with bruxism and masticatory muscle pain. After MENS application, they found repair processes in tissues at the cellular level, a reduction in pain measured on VAS (visual analogue scale), and reduction in tenderness of examined muscles using a digital pressometer. After applying TENS, repair processes were not registered, and there was a lower reduction in pain and tenderness than in the first group. According to the authors, MENS use can produce better results, especially in patients after injuries, because, in addition to analgesic effects, it can also have repairing ones. However, they pointed out the need to expand the research due to the small number of patients and lack of previous studies in that direction. They also took into account the possibility of using MENS as a complement to the use of TENS in the treatment of masticatory muscle pain in patients with bruxism.

Research on the use of TENS in patients with TMD (temporomandibular disorders) highlighted the safety of use, non-invasiveness, analgesic effect, myorelaxation, and lowered electromyographic activity [48, 49]. Besides the theory of the peripheral effect of electrotherapy, the theory of central action, i.e. the theory of the control gate, was introduced in 1965 [50]. Transcutaneous electrostimulation, similar in action to a laser, can also be used to increase the range of the opening of the jaw (in patients with that kind of dysfunction) [51].

Shanavas et al. [52] compared the use of TENS and analgesics in the treatment of pain symptoms in TMD. Studies showed that a combination of electrical stimulation and the use of analgesics and myorelaxants is more effective in treating pain than pharmaceutic therapies alone. Similar effects in the treatment of masticatory muscle pain, using only TENS, were reported by Rodrigues et al. [49], Kato et al. [53], and Moger et al. [54]. The last showed an analgesic effect mainly in the treatment of muscular and chronic pain.

Treacy [43] compared muscular awareness relaxation training (MART) and transcutaneous electrical nerve stimulation (TENS). Using MART gave an increase in the maximum range of motion while opening the mouth and reducing masseter and pterygoid muscle tension. The advantage of that technique is the involvement of the whole body (by drawing attention to posture, breathing, etc.), while TENS is a purely local action. Both those methods enabled a significant reduction in electromyographic activity. Considering all the evaluated parameters, Treacy [43] achieved significantly better results in patients treated with MART. The author emphasised the need to encourage patients to use self-regulatory therapies in order to reduce the tension of the whole body.

Muscular awareness relaxation training is performed during daily activities, by alternating tension and relaxation of muscles and picturing calming images with training diaphragmatic breathing $[35,55]$. Therapeutic exercises also have a place in the treatment of bruxism; however, most authors point to the need for further studies because of the small number of examined patients and lack of a precise description of exercises (intensity, frequency, number of repetitions, duration) [35, $51,55]$. On the basis of collected articles on the treatment of TMD (temporomandibular disorders), Moraes et al. [55] specified stretching and relaxing, coordination improvement, and strengthening and endurance increasing exercises. Stretching exercises, which may be useful in bruxism, are applied in cases of reduced movement ability in order to alleviate pain by improving local blood flow and to reduce the muscle tone [44, 56-58].

\section{PSYCHOTHERAPY}

Another important element of the multidirectional bruxism therapy is psychotherapy, which can include, among others, cognitive-behavioural therapy and biofeedback or relaxation exercises. Cognitive-behavioural therapy is a solution based on changing the pattern of behaviour, which is only possible if there is a change in the mind-set of the patient [59]. The cognitive-behavioural model is composed of many different elements, for example, relaxation, the ability to control behaviour, and various exercises to consolidate new behavioural patterns [60]. Drawbacks mentioned by Ommerborn et al. [38] are time-consumption and require long-term follow-up in order to maintain learned patterns of behaviour. When using that treatment, it is important to create the treatment protocol and then strictly monitor it. An important issue is also drawing patients' attention to nasal breathing and then shifting the patient to this type of breathing. According to Orthlieb et al. [61], no improvement after 3-6 months of starting the therapy indicates the need for re-diagnosis.

\section{BIOFEEDBACK}

Biofeedback consists of informing the patient of their body functions and convincing the patient to change their behaviour to improve health [62]. Its purpose is to 
bring about a learned response to a stimulus, e.g. visual, audible, electrical, or vibrational. When using that technique in the treatment of bruxism, a bruxism episode generates a stimulus that allows the patient to recognise and induce a conscious reaction (mainly muscle relaxation) in patients clenching their teeth during the day. During sleep, there are two types of stimuli - interrupting the continuity of sleep and not waking the patient in order to trigger a subconscious response after sending a signal to the brain. Previous studies indicated that biofeedback cannot be based on a sleep interrupting stimulus. The stimulus in the form of CES (contingent electrical stimulation) may also cause local effects, including biochemical changes [63, 64]. Disadvantages of that type of treatment may comprise increasing fatigue and daytime sleepiness as a result of waking during night rest [65]. In addition, an electrical stimulus, if its parameters are improperly set, may cause pain. Other stimuli may also be experienced as unpleasant [63]. So far, the therapeutic value of biofeedback in the treatment of bruxism has not been fully recognised [66]. It is a method that is expected to produce permanent reduction in the severity of bruxism, but this has not been confirmed yet [46].

Shedden Mora et al. [67] compared biofeedbackbased cognitive-behavioural therapy (BFB-CBT) to standard splint therapy. The use of CBT and biofeedback aims to increase awareness of parafunctions, reducing muscle tension and severity of bruxism [68]. Studies showed a (statistically and clinically) significant improvement in terms of pain intensity and pain-related disability in patients undergoing splint therapy and in those undergoing BFB-CBT, but the latter group was more satisfied with the overall effect of the treatment. Those patients also showed greater ability to cope with pain. When comparing occlusal splints and BFB-CBT, according to the authors, they proved to be equally effective in the treatment of pain [67]. All authors clearly indicated the need for further research in that direction.

\section{PHARMACOLOGICAL TREATMENT}

Pharmacological treatment can be used as a complement to the treatment of effects of intense, very severe bruxism. Some of the used drugs are: dopamine agonists, anxiolytics, buspirone, non-benzodiazepine hypnotics, antiepileptic drugs, or botulinum toxin [69-72].

Huynh et al. [73] compared propranolol and clonidine use in the treatment of nocturnal bruxism. The use of propranolol did not significantly influence the frequency and severity of bruxism compared to the placebo group. The group receiving treatment with clonidine showed more than $60 \%$ reduction in the frequency of bruxism episodes. In both groups, no difference in questionnaires filled out after waking up was noticed. Both drugs may cause prolonged morning pressure reduction and pressure reduction during the day in some patients, hence the use of those drugs in patients with low morning pressure should be carefully considered. Another side effect of using clonidine is dry mouth felt after waking up.

Studies conducted by Moosavi et al. [74] on experimental trihexyphenidyl (anticholinergic, THP) use in the treatment of bruxism were performed on four patients in whom prior treatment with other drugs resulted in no improvement. In each of those individuals, bruxism symptoms subsided after implementation of THP. One to two weeks after the end of the treatment the symptoms returned. The authors reported minor side effects compared to other commonly used drugs because of the low dose of the drug (1-2 mg THP). Due to the small group of examined patients, general conclusions could not be drawn. Many drugs previously used in the treatment of the disease proved to be ineffective in some cases, such as tricyclic antidepressants [75]. In some cases, serotonin reuptake inhibitors can cause episodes of bruxism, which can be effectively prevented by buspirone administration [76].

The use of currently popular botulinum toxin can sometimes cause serious complications and has considerable side effects, for example: alterations in saliva consistency, itching, bruising, headache, facial pain, anxiety, muscle stiffness, ringing in ears, inadvertent weakness of the facial muscles, swallowing, speech, etc. [77-80]. Injections of botulinum toxin have a short-term effect. Different results were received by Kim et al. [81] in their research on the treatment of patients with myofascial pain, trismus, and/or TMJ sounds. All patients who underwent treatment with botulinum toxin type A (BTX-A treatment) had previously been treated with physiotherapy, pharmacotherapy, behavioural therapy, or a stabilisation splint. Most of them had parafunctions such as bruxism. In that group, Kim et al. [81] received a statistically significant reduction in muscle tenderness and pain without significant adverse effects. It was possible to reduce muscle tenderness for a period of three to six months. Meunier et al. [82] also observed no significant side effects. Similar effects in reducing myofascial pain were obtained by Sidebottom et al. [83]. Guarda-Nardini et al. [84] conducted a double-blind trial on patients with bruxism or myofascial pain, using botulinum toxin in one group and a placebo in the other. When comparing the severity of pain at rest and during chewing, they noted improvement in those parameters in the Botox group. The same group also reported a slight increase in the range of motion, but no increase in mastication efficiency was registered in either of the groups.

It should be noted that most authors point to the need for further research in order to find an optimal treatment of patients with bruxism. An individual approach and therapy taking into account systemic diseases and earlier treatment should be never neglected. 


\section{CONFLICT OF INTEREST}

The authors declare no potential conflicts of interest with respect to the research, authorship, and/or publication of this article.

\section{References}

1. Lobbezoo F, Ahlberg J, Glaros AG, et al. Bruxism defined and graded: an international consensus. J Oral Rehabil 2013; 40: 2-4.

2. Behr M, Hahnel S, Faltermeier A, et al. The two main theories on dental bruxism. Ann Anat 2012; 194: 216-219.

3. Alkan A, Bulut E, Arici S, Sato S. Evaluation of treatments in patients with nocturnal bruxism on bite force and occlusal contact area: a preliminary report. Eur J Dent 2008; 2: 276-282.

4. Gomes CAF de P, El Hage Y, Amaral AP, et al. Effects of massage therapy and occlusal splint therapy on electromyographic activity and the intensity of signs and symptoms in individuals with temporomandibular disorder and sleep bruxism: a randomized clinical trial. Chiropr Man Therap 2014; 22: 43.

5. Reddy SV, Kumar MP, Sravanthi D, et al. Bruxism: a literature review. J Int Oral Health 2014; 6: 105-109.

6. Bader G, Lavigne G. Sleep bruxism; an overview of an oromandibular sleep movement disorder. Sleep Med Rev 2000; 4: 27-43.

7. Bernhardt O, Imhoff B, Lange M, Ottl P. Bruxism: etiology, diagnosis and treatment. J Craniomand Func 2015; 7: 71-74.

8. Gomes CAF de P, El-Hage Y, Amaral AP, et al. Effects of massage therapy and occlusal splint usage on quality of life and pain in individuals with sleep bruxism: a randomized controlled trial. J Jpn Phys Ther Assoc 2015; 18: 1-6.

9. Vieira e Silva CA, da Silva MA, Melchior M de O, et al. Treatment for TD with occlusal splint and electromyographic control: application of the FARC protocol in a Brazilian population. Cranio 2012; 30: 218-226.

10. Dubé C, Rompré PH, Manzini C, et al. Quantitative polygraphic controlled study on efficacy and safety of oral splint devices in tooth-grinding subjects. J Dent Res 2004; 83: 398-403.

11. Okkerse W, Brebels A, De Deyn PP, et al. Influence of a bite-plane according to Jeanmonod, on bruxism activity during sleep. J Oral Rehabil 2002; 29: 980-985.

12. Saueressig AC, Mainieri VC, Grossi PK, et al. Analysis of the influence of a mandibular advancement device on sleep and sleep bruxism scores by means of the BiteStrip and the Sleep Assessment Questionnaire. Int J Prosthodont 2010; 23: 204-213.

13. Karakis D, Dogan A, Bek B. Evaluation of the effect of two different occlusal splints on maximum occlusal force in patients with sleep bruxism: a pilot study. J Adv Prosthodont 2014; 6: 103-108.

14. Singh PK, Alvi HA, Singh BP, et al. Evaluation of various treatment modalities in sleep bruxism. J Prosthet Dent 2015; 114: 426-431.

15. Landry ML, Rompré PH, Manzini C, et al. Reduction of sleep bruxism using a mandibular advancement device: an experimental controlled study. Int J Prosthodont 2006; 19: 549-556.

16. Wright E, Anderson G, Schulte J. A randomized clinical trial of intraoral soft splints and palliative treatment for masticatory muscle pain. J Orofac Pain 1995; 9: 192-199.

17. Pettengill CA, Growney MR Jr, Schoff R, Kenworthy CR. A pilot study comparing the efficacy of hard and soft stabilizing appliances in treating patients with temporomandibular disorders. J Prosthet Dent 1998; 79: 165-168.

18. Scopel V, Alves da Costa GS, Urias D. An electromyographic study of masseter and anterior temporalis muscles in extra-articular myogenous TMJ pain patients compared to an asymptomatic and normal population. Cranio 2005; 23: 194-203.

19. Giannakopoulos NN, Katsikogianni EN, Hellmann D, et al. Comparison of three different options for immediate treatment of painful temporomandibular disorders: a randomized, controlled, pilot trial. Acta Odontol Scand 2016; 74: 480-486.
20. Truelove E, Huggins KH, Mancl L, Dworkin SF. The efficacy of traditional, low-cost and nonsplint therapies for temporomandibular disorder: a randomized controlled trial. J Am Dent Assoc 2006; 137: 1099-1107; quiz 1169.

21. Weggen T, Schindler HJ, Kordass B. Hugger A. Clinical and electromyographic follow-up of myofascial pain patients treated with two types of oral splint: a randomized controlled pilot study. Int J Comput Dent 2013; 16: 209-224.

22. Zhang FY, Wang XG, Dong J, et al. Effect of occlusal splints for the management of patients with myofascial pain: a randomized controlled, double-blind study. Chin Med J 2013; 126: 2270-2275.

23. Magdaleno F, Ginestal E. Side effects of stabilization occlusal splints: a report of three cases and literature review. Cranio 2010; 28: 128-135.

24. Naikmasur V, Bhargava P, Guttal K, Burde K. Soft occlusal splint therapy in the management of myofascial pain dysfunction syndrome: a follow-up study. Indian J Dent Res 2008; 19: 196-203.

25. Villalón P, Arzola JF, Valdivia J, Miralles R. The occlusal appliance effect on myofascial pain. Cranio 2013; 31: 84-91.

26. Harada T, Ichiki R, Tsukiyama Y, Koyano K. The effect of oral splint devices on sleep bruxism: a 6-week observation with an ambulatory electromyographic recording device. J Oral Rehabil 2006; 33: 482-488.

27. Nascimento LL, Amorim CF, Giannasi LC, et al. Occlusal splint for sleep bruxism: an electromyographic associated to Helkimo Index evaluation. Sleep Breath 2008; 12: 275-280.

28. Dalewski B, Chruściel-Nogalska M, Frączak B. Occlusal splint versus modified nociceptive trigeminal inhibition splint in bruxism therapy: a randomized, controlled trial using surface electromyography. Aust Dent J 2015; 60: 445-454.

29. Roark Al, Glaros AG, O’Mahony AM. Effects of interocclusal appliances on EMG activity during parafunctional tooth contact. J Oral Rehabil 2003; 30: 573-577.

30. Amorim CF, Giannasi LC, Ferreira LMA, et al. Behavior analysis of electromyographic activity of the masseter muscle in sleep bruxers: J Bodyw Mov Ther 2010; 14: 234-238.

31. Fricton J. Current evidence providing clarity in management of temporomandibular disorders: summary of a systematic review of randomized clinical trial for intra-oral appliances and occlusal therapies. J Evid Based Dent Pract 2006; 6: 48-52.

32. Abekura H, Yokomura M, Sadamori S, Hamada T. The initial effects of occlusal splint vertical thickness on the nocturnal EMG activities of masticatory muscles in subjects with a bruxism habit. Int J Prosthodont 2008; 21: 116-120.

33. Baad-Hansen L, Jadidi F, Castrillon E, et al. Effects of a nociceptive trigeminal inhibitory splint on electromyographic activity in jaw closing muscles during sleep. J Oral Rehabil 2007; 34: 105-111.

34. Gawriołek K, Piotrowski P. Assessment of temporomandibular dysfunction therapy progress based upon mandibular velocity measurement. J Stoma 2012; 65: 395-403.

35. Amorim CSM, Firsoff EFO, Vieira GF, et al. Effectiveness of two physical therapy interventions, relative to dental treatment in individuals with bruxism: study protocol of a randomized clinical trial. Trials 2014; 15: 8.

36. Visscher CM, Lobbezoo F, Naeije M. Treatment of bruxism: physiotherapeutic approach. Ned Tijdschr Tandheelkd 2000; 107: 293-296.

37. Knutson GA. Vectored upper cervical manipulation for chronic sleep bruxism, headache, and cervical spine pain in a child. J Manipulative Physiol Ther 2003; 26: E16.

38. Ommerborn MA, Schneider C, Giraki M, et al. Effects of an occlusal splint compared with cognitive-behavioral treatment on sleep bruxism activity. Eur J Oral Sci 2007; 115: 7-14.

39. Rajpurohit B, Khatri SM, Metgud D, Bagewadi A. Effectiveness of transcutaneous electrical nerve stimulation and microcurrent electrical nerve stimulation in bruxism associated with masticatory muscle pain - a comparative study. Indian J Dent Res 2010; 21: 104-106.

40. Watanabe A, Kanemura K, Tanabe N, Fujisawa M. Effect of electromyogram biofeedback on daytime clenching behavior in subjects with masticatory muscle pain. J Prosthodont Res 2011; 55: 75-81. 
41. Dallanora LJ, Faltin PP, Inoue RT, Santos VM. Accupuncture use in the treatment of patients with bruxism. Rev Gaúcha Odontol 2004; 52: 333-339.

42. Quintero Y, Restrepo CC, Tamayo V, et al. Effect of awarenes through movement on the head posture of bruxist children. J Ora Rehabil 2009; 36: 18-25.

43. Treacy K. Awareness/relaxation training and transcutaneous elec trical neural stimulation in the treatment of bruxism. J Oral Rehabil 1999; 26: 280-287.

44. McNeely ML, Armijo Olivo S, Magee DJ. A systematic review of the effectiveness of physical therapy interventions for temporomandibular disorders. Phys Ther 2006; 86: 710-725.

45. Michelotti A, Steens MH, Farella M, Parisini F. Short-term effects of physiotherapy versus counselling for the treatment of myofascial pain of the jaw muscles. J Oral Rehabil 2002; 29: 874 .

46. Jadidi F, Castrillon E, Svensson P. Effect of conditioning electrical stimuli on temporalis electromyographic activity during sleep. J Oral Rehabil 2008; 35: 171-183.

47. Albertin A, Kerppers II, Amorim CF, et al. The effect of manual therapy on masseter muscle pain and spasm. Electromyogr Clin Neurophysiol 2010; 50: 107-112.

48. Núñez SC, Garcez AS, Suzuki SS, Ribeiro MS. Management of mouth opening in patients with temporomandibular disorders through low-level laser therapy and transcutaneous electrical neural stimulation. Photomed Laser Surg 2006; 24: 45-49.

49. Rodrigues D, Siriani AO, Bérzin F. Effect of conventional TENS on pain and electromyographic activity of masticatory muscles in TMD patients. Braz Oral Res 2004; 18: 290-295.

50. Santana JM, Lauretti GR. Possíveis mecanismos de ação da estim ulação elétrica nervosa transcutânea no controle da dor. Rev Dor 2006; 7: 716-728.

51. Grossmann E, Tambara JS, Grossmann TK, Tesseroli JT de S Transcutaneous electrical nerve stimulation for temporomandibular joint disorder. Rev Dor 2012; 13: 271-276.

52. Shanavas M, Chatra L, Shenai P, et al. Transcutaneous electrica nerve stimulation therapy: An adjuvant pain controlling modality in TMD patients - A clinical study. Dent Res J 2014; 11: 676-679.

53. Kato MT, Kogawa EM, Santos CN, Conti PCR. TENS and lowlevel laser therapy in the management of temporomandibular disorders. J Appl Oral Sci 2006; 14: 130-135.

54. Moger G, Shashikanth MC, Sunil MK, Shambulingappa P. Transcutaneous electrical nerve stimulation therapy in temporomandibular disorder: A clinical study. J Indian Acad Oral Med Radiol 2011; 23: 46-50

55. Moraes Ada R, Sanches ML, Ribeiro EC, Guimarães AS. Therapeutic exercises for the control of temporomandibular disorders. Dental Press J Orthod 2013; 18: 134-139.

56. Clark GT. Classification, causation and treatment of masticatory myogenous pain and dysfunction. Oral Maxillofac Surg Clin North Am 2008; 20: 145-157.

57. De Leeuw R (ed.). Orofacial pain: Guidelines for assessment. Diagnosis and management. $4^{\text {th }}$ ed. Quintessence, Chicago 2008, 179-185.

58. Fricton J. Myogenous temporomandibular disorders: diagnostic and management considerations. Dent Clin North Am 2007; 51 61-83.

59. Fjellström M, Yakob M, Söder B. A modified cognitive behavioural model as a method to improve adherence to oral hygiene instructions - a pilot study. Int J Dent Hyg 2010; 8: 178-182.

60. Ohrbach R. Behavioral therapy in TMDs: an evidence based ap proach to diagnosis and treatment. Laskin DM, Greene C, Hylander W (eds.). Temporomandibular Disorders: An Evidence-Based Approach to Diagnosis and Treatment. Quintessence, Chicago 2006; 391-402.

61. Orthlieb JD, Tran TNN, Camoin A, Mantout B. Propositions for a cognitive behavioral approach to bruxism management. J Stomat Occ Med 2013; 6: 6-15.

62. Frank DL, Khorshid L, Kiffer JF, et al. Biofeedback in medicine: who, when, why and how? Ment Health Fam Med 2010; 7: 85-91.
63. Ilovar S, Zolger D, Castrillon E, et al. Biofeedback for treatment of awake and sleep bruxism in adults: systematic review protocol. Syst Rev 2014; 3: 42.

64. Molina OF, Santos ZC, Scotta P, et al. Somatisation and dissociation: a comparison study in bruxers subgroups. Rev Neuroscienc 2013; 21: 77-84.

65. Wieselmann-Penkner K, Janda M, Lorenzoni M, Polansky R. A comparison of the muscular relaxation effect of TENS and EMG-biofeedback in patients with bruxism. J Oral Rehabil 2001; 28: 849-853.

66. Lobbezoo F, van der Zaag J, van Selms MK, et al. Principles for the management of bruxism. J Oral Rehabil 2008; 35: 509-523.

67. Shedden Mora MC, Weber D, Neff A, Rief W. Biofeedbackbased cognitive-behavioral treatment compared with occlusal splint for temporomandibular disorder: a randomized controlled trial. Clin J Pain 2013; 29: 1057-1065.

68. Glaros AG. Temporomandibular disorders and facial pain: a psychophysiological perspective. Appl Psychophysiol Biofeedback 2008; 33: 161-171.

69. Veiga N, Ângelo T, Ribeiro O, Baptista A. Bruxism - literature review. Int J Dent Oral Health 2015; doi: http://dx.doi.org/ 10.16966/2378-7090.134.

70. Strausz T, Ahlberg J, Lobbezoo F, et al. Awareness of tooth grinding and clenching from adolescence to young adulthood: a nineyear follow-up. J Oral Rehabil 2010; 37: 497-500.

71. Sutin AR, Terracciano A, Ferrucci L, Costa PT. Teeth grinding: is emotional stability related to bruxism? J Res Pers 2010; 44: 402-405.

72. Tsai CM, Chou SL, Gale EN, McCall WD Jr. Human masticatory muscle activity and jaw position under experimental stress. J Oral Rehabil 2002; 29: 44-51.

73. Huynh N, Lavigne GJ, Lanfranchi PA, et al. The effect of 2 sympatholytic medications - propranolol and clonidine - on sleep bruxism: experimental randomized controlled studies. Sleep 2006; 29: 307-316

74. Moosavi SM, Setareh J, Ahmadi M, et al. Treatment of bruxism with trihexiphenidyl, a case series. Am J Psych Neurosc 2015; 3: 108-110.

75. Raigrodski AJ, Christensen LV, Mohamed SE, Gardiner DM. The effect of four-week administration of amitriptyline on sleep bruxism. A double-blind crossover clinical study. Cranio 2001; 19: $21-25$

76. Sabuncuoglu O, Ekinci O, Berkem M. Fluoxetine-induced sleep bruxism in an adolescent treated with buspirone: a case report. Spec Care Dentist 2009; 29: 215-217.

77. Lu DW, Lippitz J. Complications of botulinum neurotoxin. Dis Mon 2009; 55: 198-211.

78. Archana MS. Toxin yet non toxic: botulinum toxin in dentistry. Saudi Dent J 2016; 28: 63-69.

79. Dressler D, Benecke R. Autonomic side effects of botulinum toxin type B treatment of cervical dystonia and hyperhidrosis. Eur Neurol 2003; 49: 34-38.

80. Srivastava S, Kharbanda S, Pal US, Shah V. Applications of botulinum toxin in dentistry: a comprehensive review. Natl J Maxillofac Surg 2015; 6: 152-159.

81. Kim HS, Yun PY, Kim YK. A clinical evaluation of botulinum toxin - a injections in the temporomandibular disorder treatment. Maxillofac Plast Reconstr Surg 2016; 38: 5.

82. Meunier FA, Schiavo G, Molgó J. Botulinum neurotoxins: from paralysis to recovery of functional neuromuscular transmission. J Physiol Paris 2002; 96: 105-113.

83. Sidebottom AJ, Patel AA, Amin J. Botulinum injection for the management of myofascial pain in the masticatory muscles. A prospective outcome study. Br J Oral Maxillofac Surg 2013; 51: 199-205.

84. Guarda-Nardini L, Manfredini D, Salamone M, et al. Efficacy of botulinum toxin in treating myofascial pain in bruxers: a controlled placebo pilot study. Cranio 2008; 26: 126-135. 